\title{
TEST RESULTS OF A FAST BEAM CHOPPER WITH MA CORES
}

\author{
Y. Mori, M. Muto, C. Ohmori, $\underline{\text { Y. Shirakabe }}^{1}$, A. Takagi, S. Yamaguchi' ${ }^{2}$ KEK, Tsukuba, Japan
}

\section{Abstract}

A new type of fast beam chopper was developed and installed at the HIMAC facility. Test operations of the chopper have been carried out. The chopper is basically a periodic beam-energy transformer that performs $10 \%$ modulation of beam's kinetic energy. The modulated fraction of the beam is chopped off by the downstream RFQ due to the relatively narrow injection energy window of the RFQ.

Some of the recent test results with the chopper are presented. Measured chopped beams show sharp spikes at the pulse edges. Their origin is studied by numerical calculations. Another topic is the beam injection studies at the HIMAC's main-ring with the use of the chopped beams.

\section{INTRODUCTION}

An R \& D work for developing an efficient beam chopper for high-intensity proton accelerators has been on the way. The principal demand is to achieve a clear chopping with high intensity beams within a minimal installation length in the beam course.

The chopper we present here is of a beam-energy transformer type[1]. An inductive voltage is generated periodically on the beam axis that modulates beam's kinetic energy by $10 \%$. Since the downstream RFQ cannot accelerate the modulated fraction of the beam due to its narrow energy window[2], chopping off of the beam is effectively achieved with this combination.

The beam chopper based on the above principle was developed and installed at HIMAC, the heavy ion medical accelerator facility for cancer therapy in Chiba, Japan. Test operations and experiments using this chopper have been carried out. Some of the obtained results are presented.

\section{CHOPPING EXPERIMENTS}

As was shown in our previous report[2], chopped beam pulses show sharp spikes. Although the chopping is effectively done both in the lower-side modulation and in the higher-side modulation, the spike positions are different in both cases, namely at the front-edge of the pulse in the former case, and at the rear-edge in the latter.

This is caused by the finite rise and fall speed of the chopper voltage (Figure 2). When the chopper voltage is rising toward the beam's downstream direction, latecoming particles are more accelerated than earlier ones, causing bunching of the beam intensity. And at the falling

\footnotetext{
1 yoshihisa.shirakabe@kek.jp

2 Present address: Chiba University, 1-33, Yayoi-cho, Inage-ku, Chiba, 263-8522 Japan
}

of the chopper voltage, there occurs debunching.

In the lower-side modulation (top), the ion beam energy reaches the linac's injection energy, $8 \mathrm{keV} / \mathrm{u}$, at the end of the chopper-voltage rise, where the chopped pulse starts. Thus the spike caused by the bunching appears at the start point of the pulse. In the higher-side modulation (bottom), contrary to the former case, the voltage rise starts at the end of the chopped pulse, where the spike appears.

Numerical calculations were carried out to visualize this process[3]. The results are shown in Figure 3 in comparison with the measurements. In this calculation, the chopper voltage was assumed to rise and fall in $50 \mathrm{nsec}$ in sinusoidal curves, roughly simulating the observed chopper voltage[2]. The difference of the particles' speed caused by the beam-energy modulation is reflected to the difference of the current density. As is seen from Figure 3, this simplified model is qualitatively sufficient to show that the finite rise and fall speed of the chopper voltage are the origin of the spikes.

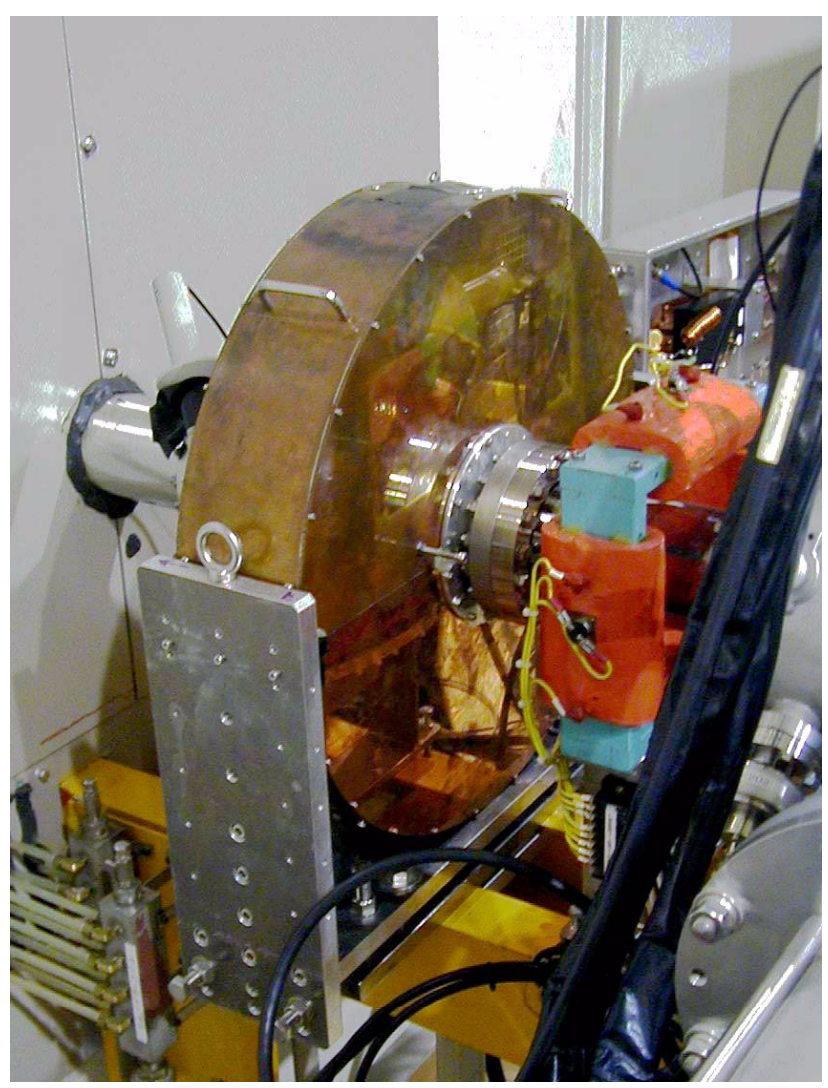

Figure 1: Chopper cavity installed at the HIMAC facility. A constant-energy beam from an ion source comes from the right-hand side, and its energy is modulated by $+-5 \%$ at the chopper cavity. The downstream RFQ is located at the left-hand side, which is at the other side of the wall. 
Lower-side modulation

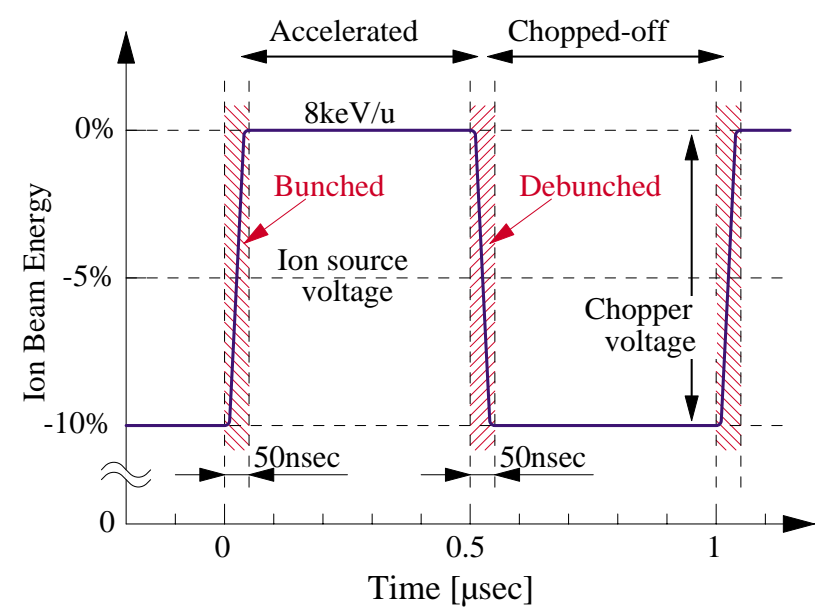

Higher-side modulation

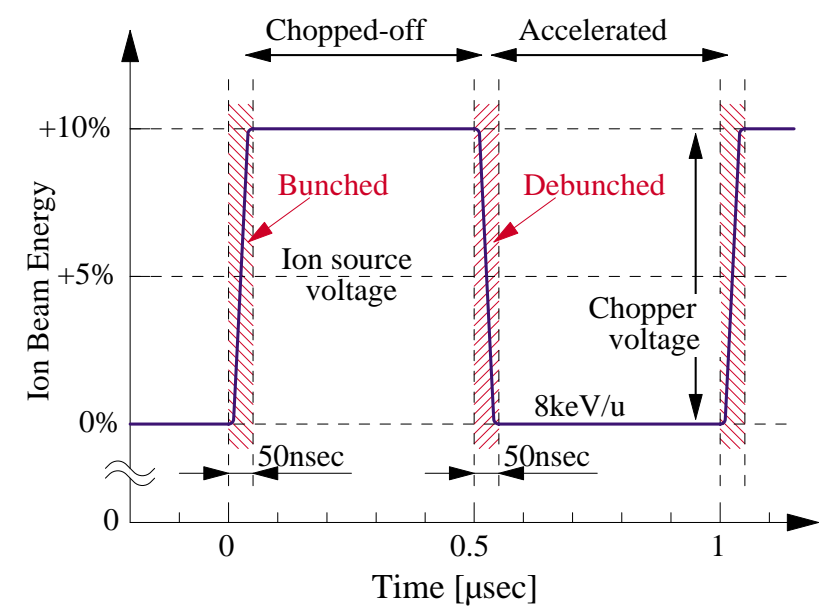

Figure 2: Simplified scheme of beam-energy modulation by the chopper voltage. The chopping is achieved both in the lower- and higher-side modulation. The chopper voltage is assumed to rise and fall in 50nsec in sinusoidal curves. In both cases of the modulation, the beam bunching occurs at the voltage rise, and the debunching at the voltage fall.

The measured waveforms in Figure 3 contain higherorder frequency components. These components make a certain part of possible origins of the difference between the measurement and the calculation. In Figure 4, an FFT spectrum of a measured chopped beam in the lower-side modulation is shown. The chopping frequency is $1 \mathrm{MHz}$ and the duty factor is $50 \%$. A sharp peak at $100 \mathrm{MHz}$ is originated from the RFQ's frequency.

\section{MAIN-RING INJECTION STUDY}

Beam injection studies at the HIMAC main-ring with the chopped beams have been carried out. If the bunch length is short enough compared to the ring's bucket size, it becomes possible to observe the bunch motion inside the bucket. Figure 5 shows some of the observed results.
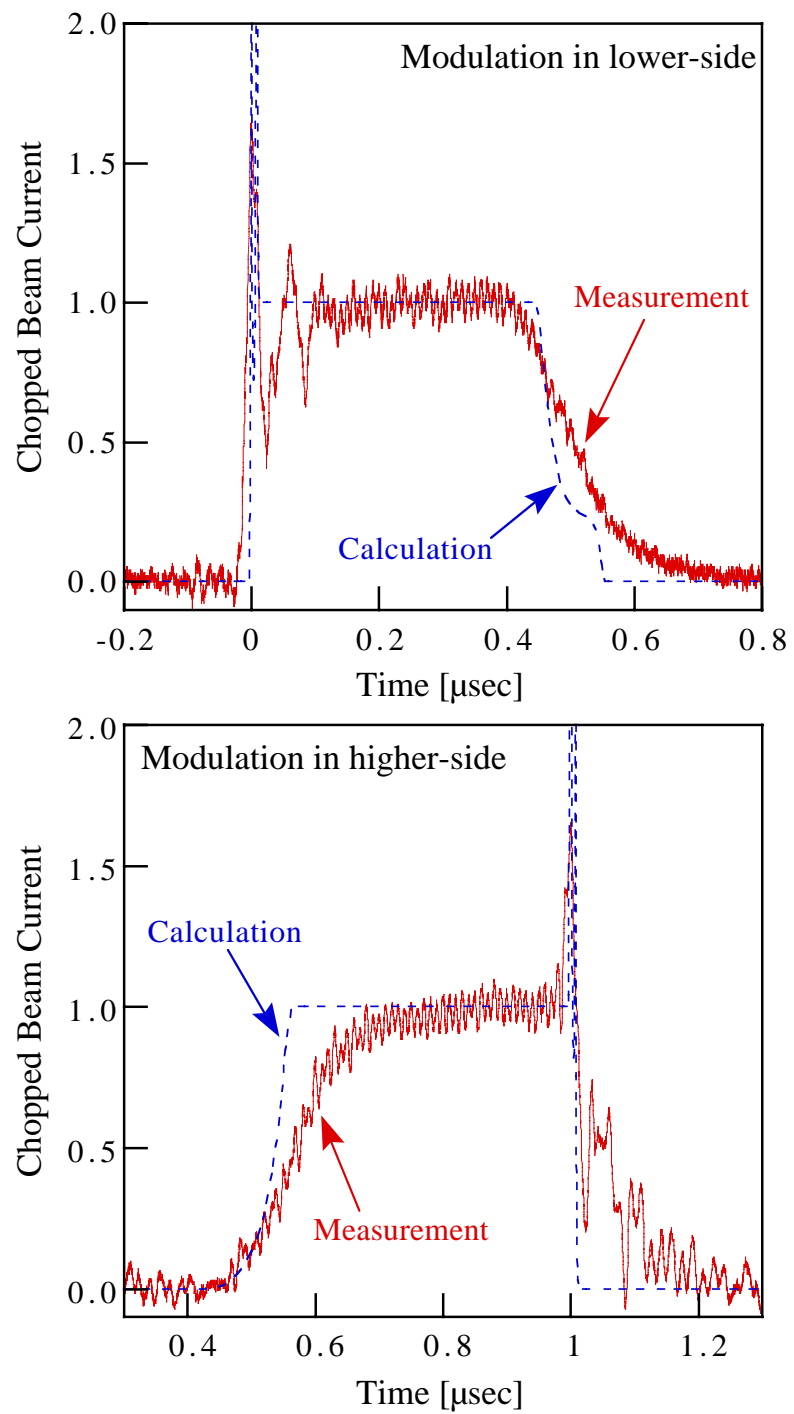

Figure 3: Measured and calculated results of chopped beam currents. When the chopping is done with the lowerside modulation, the spike appears at the front edge of the pulse (top), and with the higher-side modulation, at the rear edge (bottom). These spikes are caused with the bunching of beams due to the finite rise and fall speed of the chopper voltage. Numerical calculation was carried out, with a simplified assumption of a sinusoidal voltage rise- and fall-time of 50nsec (broken lines).

Both the top and bottom figures of Fig. 5 are the mountain range plots of the main-ring's beam signals. Each single line in the two figures denotes observed beam pulses. The lines are plotted upward. The ring has a harmonic number of 4 , and the RF of $1.04 \mathrm{MHz}$ at the injection. In normal operations, the beam is injected into the main-ring through an adiabatic capture.

The beams are chopped, prior to the injection, to make short bunches of $150 \mathrm{nsec}$ width. In the top figure, the beam is injected into all 4 buckets of the main-ring with the repetition rate of $1.04 \mathrm{MHz}$. The pulses appear in synchronization with the ring's RF. In the bottom figure, the same short bunches are injected with the repetition 

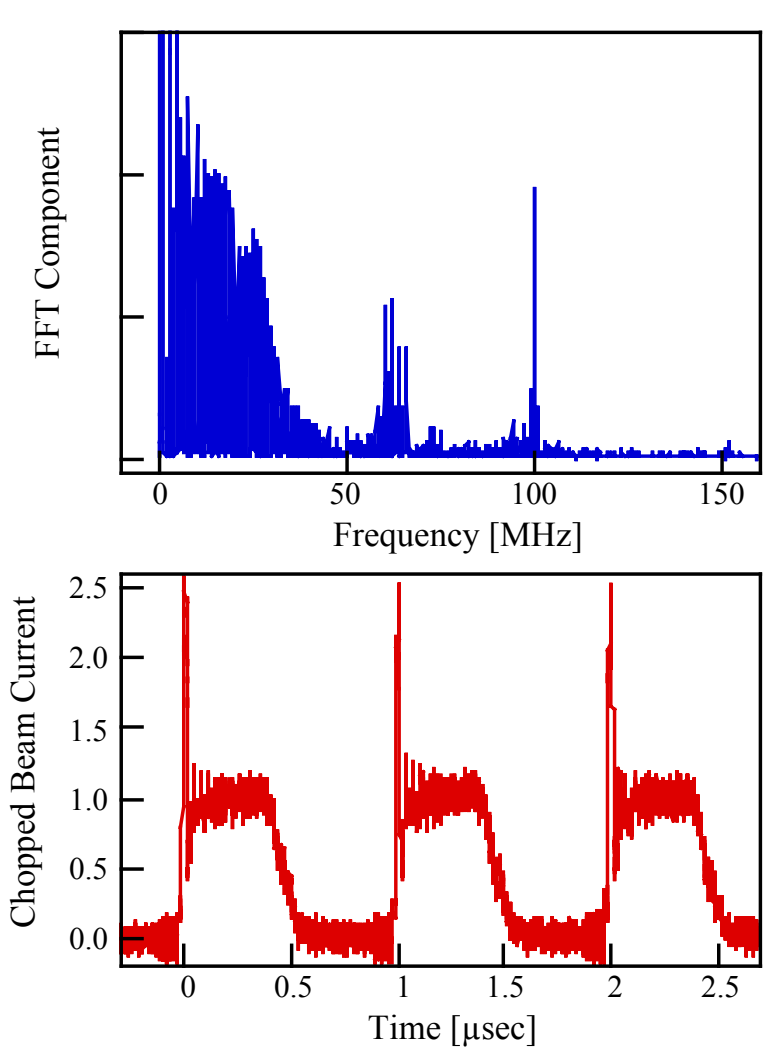

Figure 4: Fourier-transformed beam spectrum (top) with its original waveform (bottom) in the case of the lowerside modulation. The chopping frequency is $1 \mathrm{MHz}$ and the duty factor is $50 \%$.

rate of $260 \mathrm{kHz}$ to make a single bunch beam with a harmonic number of 1 . Consequently, the pulses appear only once out of 4 buckets.

In both cases, it is observed that the pulses start expanding in the longitudinal direction after $\sim 30 \mathrm{msec}$ from the injection, which is caused by the slight frequency error of the RF. In usual main-ring operations without beam chopping, such a growth cannot be observed because of the large emittance of the beams.

\section{SUMMARY}

The new type of chopper was developed and installed at the HIMAC facility. The chopper is in principle a beam energy modulator that utilizes the narrow injection energy window of the downstream RFQ. Test operations of the chopper are now on the way.

The numerical and experimental studies to understand the origin of sharp spikes of the chopped beams were presented. At the HIMAC main-ring, injection studies with short-bunch beams made by the chopper were carried out.

\section{ACKNOWLEDGEMENTS}

The authors are deeply thankful to Dr. K. Noda, Dr. M. Kanazawa, and other HIMAC staffs for their thorough assistance in the installation of the chopper system and in the following chopper experiments.
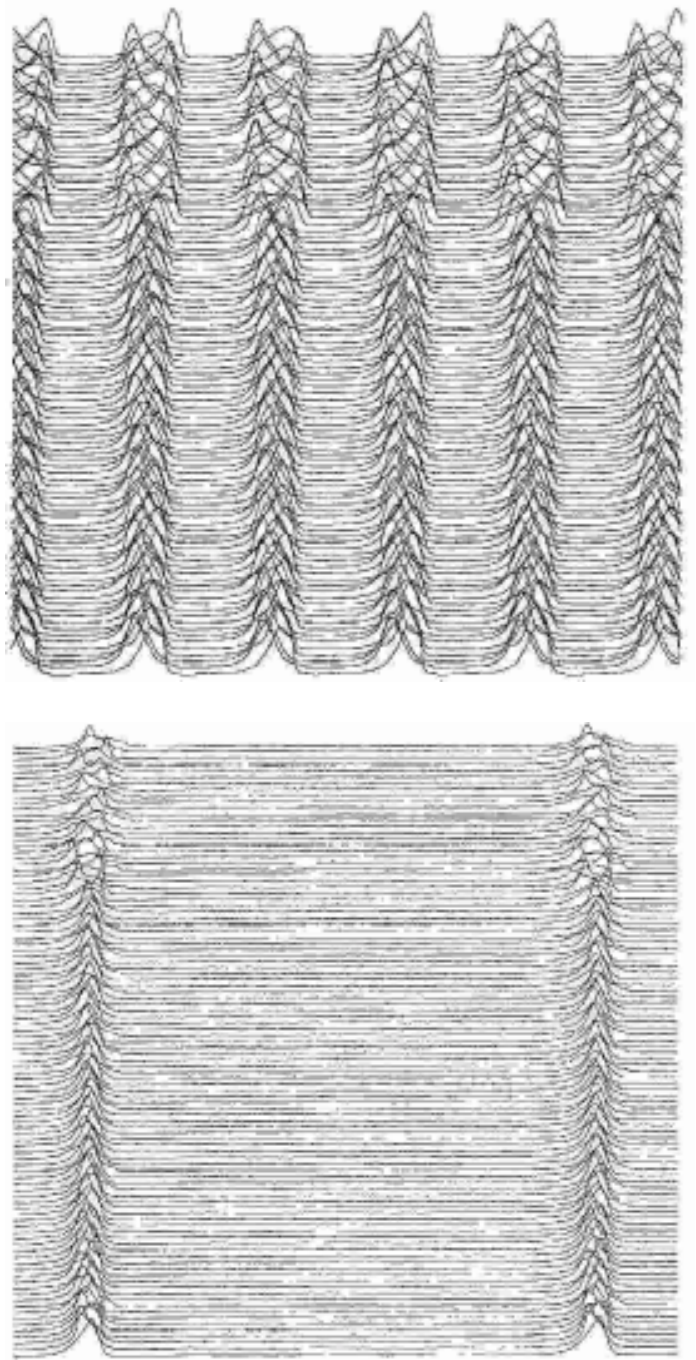

Figure 5: Captured beam profiles observed at the HIMAC main-ring. Prior to the injection, the beams are chopped to make short bunches of $150 \mathrm{nsec}$ width. In the top figure, the beam is injected into 4 buckets of the main-ring. In the bottom figure, the same short bunches are injected with a harmonic number of 1 . In both cases, the beams start growing in the longitudinal direction after $\sim 30 \mathrm{msec}$ from the injection.

\section{REFERENCES}

[1] W. Chou et al, Proc. 1999 Part. Accel. Conf., 565(1999).

[2] Y. Shirakabe et al, Proc. 2000 Euro. Part. Accel. Conf., 2468(2000).

[3] S. Yamaguchi, Master thesis, Feb. 2001, in Japanese. 